\title{
Clipping of posteriorly projecting large posterior communicating aneurysm via transsylvian anterior temporal approach
}

\author{
Junichi Yamamoto M.D., Ph.D. \\ Department of Neurosurgery, Albany Medical Center, Albany, New York
}

Clipping of a posterior projecting large posterior communicating aneurysm via transsylvian anterior temporal approach is presented. To expose and clip this large aneurysm, retraction of the temporal lobe is required via the conventional pterional approach. In this video, we demonstrate microsurgical techniques as to how to dissect and mobilize the sylvian veins as well as a bridging vein to the sphenoparietal sinus, and the middle cerebral artery branches to the frontal lobe side. After the microsurgical dissection, a wide anteriolateral temporal space is obtained without significant temporal lobe retraction. The aneurysm was completely obliterated via this approach.

The video can be found here: http://youtu.be/vU9Nxm7Yn-A.

KEYWORDS anterior temporal approach; posterior projecting internal carotid aneurysm; surgical clipping; video 